\title{
Erratum: Frequency shift between coherent superposition states induced by the Berry phase evolving linearly in time [Phys. Rev. A 92, 013403 (2015)]
}

\author{
Atsuo Morinaga and Yasushi Hasegawa \\ (Received 11 April 2016; published 23 May 2016)
}

DOI: 10.1103/PhysRevA.93.059902

In Fig. 5 and Sec. III, we misunderstood the frequency shift of the applied radio-frequency (rf) field for the shift of the frequency separation between the $|F=1, m=-1\rangle$ and $\left|F^{\prime}=2, m^{\prime}=1\right\rangle$ states under the rotation of the direction of the magnetic field with frequency $f$. The primary resonance occurs by the two-photon $\sigma^{+}$-polarized microwave and $\sigma^{+}$-polarized rf transition, while the sideband resonance with $n= \pm 2$ occurs by the four-photon transition with a $\pi$-polarized microwave, a $\pi$-polarized rf, and two $\sigma^{+}-\sigma^{+}$rotation fields with frequency $f$, as shown in Fig. 1 of this Erratum. The polarization of the microwave changes from $\sigma^{+}$to $\pi$ as an angle $\theta$ between the direction of the magnetic field and the rotation axis ( $z$ axis) rotates from 0 to $\pi / 2$. The sideband resonance with $n=-4$, which was observed at an angle $\theta$ larger than $\pi / 2$, is the same transition

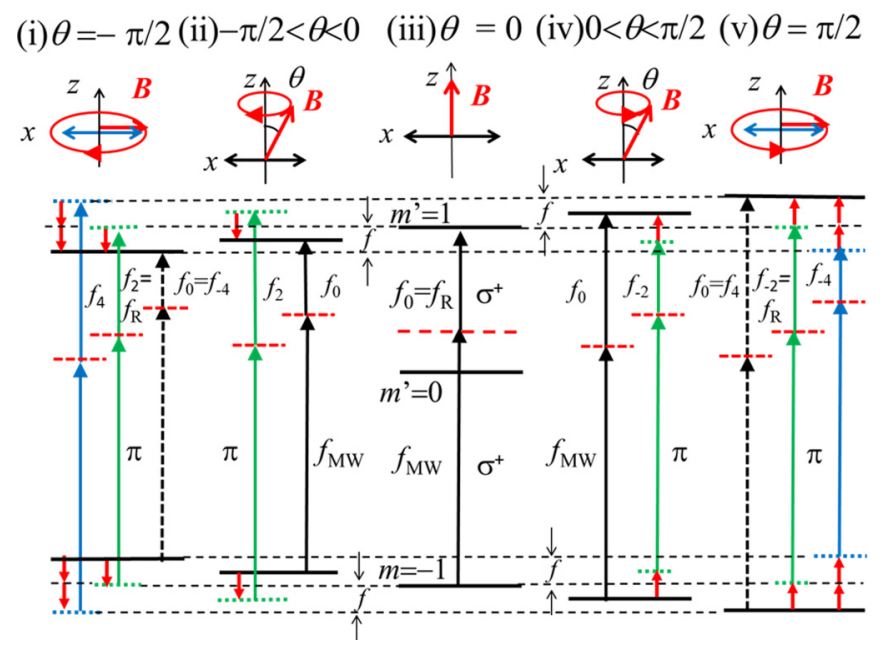

FIG. 1. Energy levels of $m=-1$ and $m^{\prime}=1$ states and multi-photon transitions for angle $\theta$ from the rotation axis ( $z$-axis) under the rotation of the direction of the magnetic field $\boldsymbol{B}$ at a frequency $f$. Here $f_{n}$ denotes the resonance frequency of the radio-frequency field for sideband resonance with $n$ and $f_{\mathrm{MW}}$ is the fixed microwave frequency with a linear polarization along the $x$ axis. Case (ii) is also applied for $\pi / 2<\theta<\pi$.

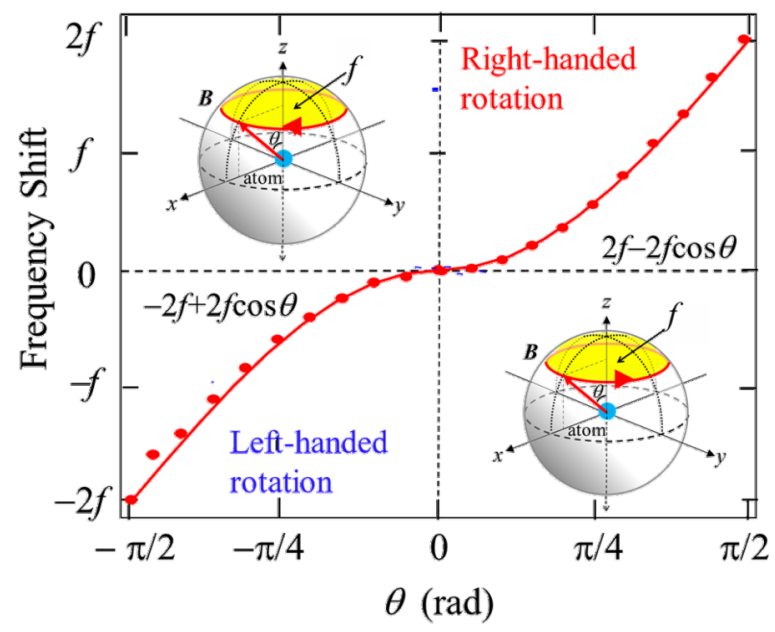

FIG. 5. Shift of the frequency separation between the $m=-1$ and $m^{\prime}=1$ states under the rotation of the magnetic field with constant frequency $f$ from that under the static magnetic field, as a function of angle $\theta$ from rotation axis ( $z$ axis). The solid curve is the time derivative of the Berry phase. 
as the primary resonance with a reversed rotation direction at angle $\pi-\theta$, because the rotation axis could be reversed to the $-z$ axis. Then the shift of the frequency separation between the $m=-1$ and $m^{\prime}=1$ states should be $f_{n}-f_{\mathrm{R}}-n f$, where $f_{n}$ and $f_{\mathrm{R}}$ are the $\mathrm{rf}$ frequency of the sideband with $n$ and that under the static magnetic field, respectively, and it is given by $-2 f+2 f \cos \theta$ in the range of $-\pi / 2 \leqslant \theta \leqslant 0$ and $2 f-2 f \cos \theta$ in the range of $0 \leqslant \theta \leqslant \pi / 2$. Therefore, the revised Fig. 5 is presented here [1].

Consequently, the abstract and conclusion also should be corrected as follows.

"The frequency shift of the spin state with $m$ under the rotation of the magnetic field with a constant frequency $f$ changes monotonically in the range of $-\pi / 2<\theta<\pi / 2$, where $\theta$ is angle from the rotation axis. It takes zero only at $\theta=0$ and two discontinuous values of $\pm m f$ at $\theta= \pm \pi / 2$. Our multiphoton spectroscopy using a magnetic-field-insensitive transition verified completely that the frequency shift is equal to the time derivative of the Berry phase $-2 m \pi(1-\cos \theta)$ for a whole rotation." We confirmed more clearly that the Berry phase depends on the spin component and the signed solid angle, but does not depend on the sign and the magnitude of the $g$-factor, as originally predicted by Berry $[2,3]$.

[1] K. Toriyama, A. Oguchi, and A. Morinaga, Phys. Rev. A 84, 062103 (2011); 93, 059903 (2016).

[2] M. V. Berry, Proc. R. Soc. London Ser. A 392, 45 (1984).

[3] S. Welte, C. Basler, and H. Helm, Phys. Rev. A 89, 023412 (2014). 